\title{
Epigenomic alterations in the individuals exposed to arsenic through drinking water in West Bengal, India
}

\author{
A.K. Giri, D. Chatterjee \& N. Banerjee \\ Molecular Genetics Division, CSIR-Indian Institute of Chemical Biology, Kolkata, India
}

\begin{abstract}
We have evaluated epigenetic changes in the arsenic exposed population at West Bengal, India. We observed that the arsenic exposed individuals had changes in the promoter methylation status of tumor suppressive and DNA repair genes. The downstream effectors mainly proteins were also altered in case of arsenic exposure. Global miRNA profiling showed several altered miRNAs in arsenic exposed samples. Of the above altered miRNAs, a significant number was associated with arsenic induced senescence and diseases like skin lesions, skin cancer, respiratory distress and peripheral neuropathy.
\end{abstract}

\section{INTRODUCTION}

Arsenic is not mutagenic but carcinogenic and human. There is a strong believe that arsenic follows the epigenetic pathways to induce cancer in humans. DNA methylation is an epigenetic modification of DNA that is tightly regulated in mammalian development and is responsible for maintaining the normal functioning of the adult organism (Schaefer et al., 2007). Micro RNAs (miRNA) are small 19-25 nucleotides long noncoding RNA molecules that functions in controlling gene expression post-transcriptionally by destabilizing the transcribed mRNA or translational repression (Filipowicz et al., 2008). Evidence showed that epigenetic modifications including DNA methylation and altered micro RNA expression patterns contribute to carcinogenesis (Watanabe et al., 2008). So here we have investigated the epigenetic alterations in arsenic exposed population in West Bengal, India.

\section{METHODS}

\subsection{Selection of study subjects and sample collection}

Study subjects were recruited from the highly arsenicaffected Murshidabad district of West Bengal. The control subjects were chosen from the arsenic unaffected district of East Midnapur. All the study subjects were age and sex matched with similar socioeconomic status. The selection criteria were followed as described in Ghosh et al. (2007). Samples were collected only from those subjects who provided informed consent to participate in the study. This study was conducted in accord with the Helsinki II Declaration and approved by the Institutional Ethics Committee of CSIR-Indian Institute of Chemical Biology. The collected samples included drinking water and urine for exposure assessment by Atomic Absorption Spectrometer and blood samples were used to isolate DNA, RNA and protein for subsequent studies.

\subsection{Identification of skin lesions and peripheral neuropathy}

An expert dermatologist identified the characteristics of arsenic-induced skin lesions and helped in the recruitment of exposed study participants. Arsenicinduced neuronal problems were recorded by an expert neurologist for the various types of clinical manifestations including the power and deep tendon reflexes, calf tenderness, pressure and pain as detailed previously (Paul et al., 2013). The studied participants, clinically confirmed to have peripheral neuropathy by the neurologist, were brought for confirmatory electrophysiological studies such as nerve conduction velocity test $(\mathrm{NCV})$ and electromyography (EMG) test.

\subsection{DNA promoter methylation, quantitative real time PCR and Western Blotting}

Briefly bisulfite modification of DNA was performed and then subjected to methylation specific PCR for the promoter regions of the three above mentioned genes separately. RNA was converted to complementary DNA (cDNA) using RevertAid H Minus First Strand cDNA Synthesis Kit and then subjected to gene or microRNA expression analysis. Protein was isolated from the peripheral blood mononuclear cells and cell culture pellet using RIPA lysis reagent and then subjected to western blot for the respected proteins.

\section{RESULTS AND DISCUSSION}

\subsection{Promoter methylation status is altered and regulates the gene expression status}

The tumor suppressor genes namely DAPK and p16 showed significant hypermethylation in the individuals with skin lesion (WSL) compared with the individuals without skin lesion (WOSL). It also shows that the individuals with DAPK and p16 hypermethylation had higher risk of developing skin lesions 


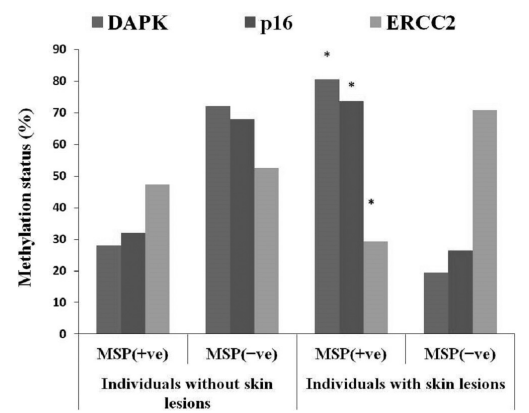

Figure 1. Promoter methylation index (\%) of DAPK, p16 and ERCC2 in human study population; ${ }^{*} \mathrm{p}<0.5$.

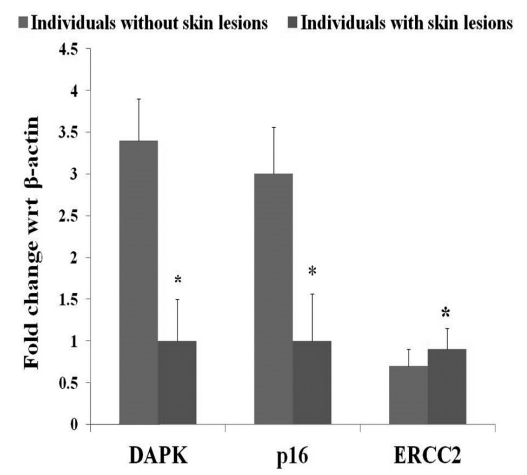

Figure 2. Alteration of protein levels in the differentially altered promoter. $* \mathrm{p}<0.5$.

as is predicted from the OR values (Banerjee et al., 2013). In humans, arsenic exposure led to hypomethylated promoters of ERCC2, and this was significantly highest in the exposed WSL compared to the referents (Fig. 1). Tumor suppressor proteins, DAPK and p16 was found to downregulated significantly whereas the DNA repair related protein i.e. ERCC2 was upregulated in the exposed population compared to the reference group (Fig. 2).

\section{2 miRNA levels are associated with arsenic induced diseases}

We performed global miRNA profiling and found that miRNA's involved in arsenic induced senescence and diseases like skin lesions and peripheral neuropathy are altered. Several senescence related miRNAs like miR-21, miR-34a, miR-29a, 126, 141 and 424 was up-regulated in arsenic exposed individuals. This suggests that epigenetic mediators like miRNAs are involved in cellular processes perturbed by arsenic. Results showed that miR-21 was up-regulated in arsenic exposed individuals and contributes to arsenic induced skin lesions, skin cancers and respiratory distress. Similarly, miR-29a was strongly associated with the occurrence of peripheral neuropathy by regulating its downstream effector PMP22 (Banerjee et al., 2017; Chatterjee et al., 2018). In vitro studies on different cell lines treated with varying doses of sodium arsenite validated the above observed results from arsenic exposed individuals.

\section{CONCLUSIONS}

Our study reveals that epigenetic mechanisms contribute to arsenic altered cellular processes like DNA repair ability, tumor suppressive mechanisms and senescence as well as diseases like skin lesions, skin cancer, respiratory distress and peripheral neuropathy. Further molecular insights into the epigenetic mechanisms might provide novel therapeutic strategies for better management of arsenic induced diseases.

\section{ACKNOWLEDGEMENTS}

The author acknowledges Council of Scientific and Industrial Research (CSIR), New Delhi, India for funding the research works and field study.

\section{REFERENCES}

Banerjee, N., Paul, S., Sau, T.J., Das, J.K., Bandyopadhyay, A., Banerjee, S. \& Giri, A.K. 2013. Epigenetic modifications of $D A P K$ and $p 16$ genes contribute to arsenic-induced skin lesions and nondermatological health effects. Toxicol. Sci. 135(2): 300-308.

Banerjee, N., Bandyopadhyay, A.K., Dutta, S., Das, J.K., Chowdhury, T.R., Bandyopadhyay, A. \& Giri, A.K. 2017. Increased microRNA 21 expression contributes to arsenic induced skin lesions, skin cancers and respiratory distress in chronically exposed individuals. Toxicology 378 : $10-16$.

Chatterjee, D., Bandyopadhyay, A., Sarma, N., Basu, S., Roychowdhury, T., Roy, S.S. \& Giri, A.K. 2018. Role of microRNAs in senescence and its contribution to peripheral neuropathy in the arsenic exposed population of West Bengal, India. Environ. Pollut. 233: 596-603.

Filipowicz, W., Bhattacharyya, S.N. \& Sonenberg, N. 2008. Mechanisms of posttranscriptional regulation by microRNAs: are the answers in sight? Nat. Rev. Genet. 9(2): 102.

Ghosh, P., Banerjee, M., De Chaudhuri, S., Chowdhury, R., Das, J.K., Mukherjee, A., Sarkar, A.K., Mondal, L., Baidya, K. \& Sau, T.J. 2007. Comparison of health effects between individuals with and without skin lesions in the population exposed to arsenic through drinking water in West Bengal, India. J. Exp. Sci. Environ. Epidemiol. 17(3): 215-223.

Paul, S., Das, N., Bhattacharjee, P., Banerjee, M., Das, J.K., Sarma, N., Sarkar, A., Bandyopadhyay, A.K., Sau, T.J. \& Basu, S. 2013. Arsenic-induced toxicity and carcinogenicity: a two-wave cross-sectional study in arsenicosis individuals in West Bengal, India. J. Exp. Sci. Environ. Epidemiol. 23(2): 156-162.

Schaefer, C.-B., Ooi, S.-K., Bestor, T.-H. \& Bourc'his, D. 2007. Epigenetic decisions in mammalian germ cells. Science. 316(5823): 398-399.

Watanabe, M., Ogawa, Y., Itoh, K., Koiwa, T., Kadin, M.E., Watanabe, T., Okayasu, I., Higashihara, M. \& Horie, R. 2008. Hypomethylation of CD30 CpG islands with aberrant JunB expression drives CD30 induction in Hodgkin lymphoma and anaplastic large cell lymphoma. Lab. Invest. 88(1): 48-57. 